\title{
Calcium window currents, periodic forcing, and chaos: Understanding single neuron response with a discontinuous one-dimensional map
}

\author{
J. Laudanski, ${ }^{1,2}$ C. Sumner, ${ }^{2}$ and S. Coombes ${ }^{1}$ \\ ${ }^{1}$ School of Mathematical Sciences, University of Nottingham, Nottingham NG7 2RD, United Kingdom \\ ${ }^{2}$ MRC Institute of Hearing Research, Science Road, Nottingham NG7 2RD, United Kingdom
}

(Received 26 April 2010; published 26 July 2010)

\begin{abstract}
Thalamocortical (TC) neurones are known to express the low-voltage activated, inactivating $\mathrm{Ca}^{2+}$ current $I_{\mathrm{T}}$. The triggering of this current underlies the generation of low threshold $\mathrm{Ca}^{2+}$ potentials that may evoke single or bursts of action potentials. Moreover, this current can contribute to an intrinsic slow $(<1 \mathrm{~Hz})$ oscillation whose rhythm is partly determined by the steady state component of $I_{\mathrm{T}}$ and its interaction with a leak current. This steady state, or window current as it is so often called, has received relatively little theoretical attention despite its importance in determining the electroresponsiveness and input-output relationship of TC neurones. In this paper, we introduce an integrate-and-fire spiking neuron model that includes a biophysically realistic model of $I_{\mathrm{T}}$. We briefly review the subthreshold bifurcation diagram of this model with constant current injection before moving on to consider its response to periodic forcing. Direct numerical simulations show that as well as the expected mode-locked responses there are regions of parameter space that support chaotic behavior. To reveal the mechanism by which the window current generates a chaotic response to periodic forcing we consider a piecewise linear caricature of the dynamics for the gating variables in the model of $I_{\mathrm{T}}$. This model can be analyzed in closed form and is shown to support an unstable set of periodic orbits. Trajectories are repelled from these organizing centers until they reach the threshold for firing. By determining the condition for a grazing bifurcation (at the border between a spiking and nonspiking event) we show how knowledge of the unstable periodic orbits (existence and stability) can be combined with the grazing condition to determine an effective one-dimensional map that captures the essentials of the chaotic behavior. This map is discontinuous and has strong similarities with the universal limit mapping in grazing bifurcations derived in the context of impacting mechanical systems [A. B. Nordmark, Phys. Rev. E 55, 266 (1997)].
\end{abstract}

DOI: 10.1103/PhysRevE.82.011924

PACS number(s): 87.19.11, 87.10.-e, 82.40.Bj

\section{INTRODUCTION}

The thalamus is often regarded as a relay station or gateway for the passage of information from the sensory periphery to the cortex. It is also known to play an important role in regulating states of sleep and wakefulness [1]. Thalamocortical (TC) relay neurones of the thalamus typically fire in one of two modes-tonic spiking or burst spiking. In the latter case the response properties of TC cells are dominated by a low threshold, transient $\mathrm{Ca}^{2+}$ current known as $I_{\mathrm{T}}$. When this current is evoked, $\mathrm{Ca}^{2+}$ entering the neuron via $T$-type $\mathrm{Ca}^{2+}$ channels causes a large voltage depolarization known as the low-threshold $\mathrm{Ca}^{2+}$ spike (LTS). Conventional action potentials mediated by fast $\mathrm{Na}^{+}$and $\mathrm{K}^{+}$(delayed-rectifier) currents often ride on the crest of an LTS resulting in a burst response (i.e., a tight cluster of spikes) $[2,3]$. As well as playing a role in burst firing, recent work on thalamic slices has shown that the $I_{\mathrm{T}}$ current can lead to membrane potential bistability, and play a role in the generation of slow $(<1 \mathrm{~Hz})$ thalamic rhythms [4-7]. This membrane potential bistability is due to an imbalance between a hyperpolarizing leak current and a depolarizing steady calcium current. This steady current occurs when there is an overlap or window in the voltage region of activation and deinactivation of $T$ channels, so that a fraction of the channels are always open and do not inactivate. Since the amplitude of this sustained current can significantly diminish as the membrane voltage moves outside of the window one might expect the creation of complex behaviors for input currents that cause substantial variations in the cell membrane potential in and out of this window.
This is one of the questions we address in this paper with a study of a model for $I_{\mathrm{T}}$.

Several conductance based models of spiking neurons with an $I_{\mathrm{T}}$ current already exist in the literature, such as those in [8-10]. Although providing a good fit to physiological data these models suffer from the same problems as the fundamental Hodgkin-Huxley model on which they are based. Namely they are high dimensional and nonlinear and not amenable to straight-forward mathematical analysis (at least not without invoking some special singular limit). In an attempt to alleviate this problem Smith et al. [11] have developed a minimal integrate-and-fire-or-burst (IFB) model that replaces the fast spiking currents with an integrate and fire mechanism. Moreover, they also caricature the $I_{\mathrm{T}}$ current with a single gating variable in such a way that it is still able to accurately reproduce data from sinusoidally forced TC neurons in cat dorsal lateral geniculate nucleus. However, their particular model for activation and inactivation precludes a window current and bistability and it is not able to produce slow intrinsic oscillations. Despite this the model does lend itself to detailed theoretical studies at both the single neuron [12-14] and network level [15-17]. In this paper we take a step back from the standard IFB model and first consider a biophysically realistic model of $I_{\mathrm{T}}$. In Sec. II, numerical bifurcation techniques are used to flesh out the behavior of subthreshold (nonspiking) dynamics in response to constant current injection. This nicely highlights that the mechanism for low frequency oscillations is a direct consequence of the window current. Indeed the presence of a sub- 
critical Hopf bifurcation can destabilize one of the fixed points and lead to a low frequency subthreshold limit cycle. The spiking dynamics is explored with direct numerical simulations in response to periodic forcing and show modelocking responses reminiscent of that already observed for the standard IFB model. However, the full $I_{\mathrm{T}}$ model has more exotic features indicative of chaotic behavior. To establish chaos, we develop a notion of Lyapunov exponents that allows us to accommodate an integrate-and-fire reset mechanism. Next in Sec. III, we show how a piecewise linear (PWL) caricature of the activation and inactivation curves of the biophysical model for $I_{\mathrm{T}}$ is sufficient to capture the essential subthreshold, spiking dynamics and bifurcations of the full model as well as its inherent bistability. Importantly trajectories of this new model can be calculated in closed form. Since numerical simulations suggest the presence of unstable orbits as organizing centers for the chaotic response to periodic forcing we focus on the analytical construction and stability analysis of periodic spiking orbits. Moreover, important dynamic transitions occur as a trajectory just reaches the threshold for firing, which motivates a further calculation of nonsmooth bifurcations associated with socalled grazes. We show that knowledge of the unstable periodic orbits and the conditions for a graze are essential ingredients for constructing a one-dimensional map that can generate chaotic dynamics of the type seen in the full threedimensional nonautonomous nonsmooth model. Finally, in Sec. IV, we discuss the relationship of the one-dimensional map to the universal limit mapping in grazing bifurcations derived in the context of impacting mechanical systems by Nordmark [18].

\section{MODEL}

Following standard practice for the description of conductance-based single neuron models we write the evolution for the membrane voltage, $v$, for a TC neuron using a current balance equation of the form:

$$
C \frac{d v}{d t}=I_{\mathrm{L}}+I+I_{\mathrm{T}}+I_{\mathrm{Sp}}
$$

Here, $C$ is the membrane capacitance, $I_{\mathrm{L}}$ a leak current of the form $g_{\mathrm{L}}\left(v_{\mathrm{L}}-v\right)$ with $g_{\mathrm{L}}$ the leak conductance and $v_{\mathrm{L}}$ the leak reversal potential, $I$ represents an injected current and the ionic current $I_{\mathrm{Sp}}$ represents the sodium and potassium currents involved in spike generation modeled with say the usual Hodgkin-Huxley form [19]. The low-threshold $T$-type calcium current $I_{\mathrm{T}}$ is a depolarizing inactivating current which is modeled as in the work of Williams et al. [4]:

$$
I_{\mathrm{T}}=g_{\mathrm{Ca}} m^{3} h\left(v_{\mathrm{Ca}}-v\right),
$$

where $g_{\mathrm{Ca}}$ and $v_{\mathrm{Ca}}$ are the conductance and reversal potential for the current, and $m$ and $h$ represent activating and inactivating gating variables. The dynamics for the gates can be written in standard form with
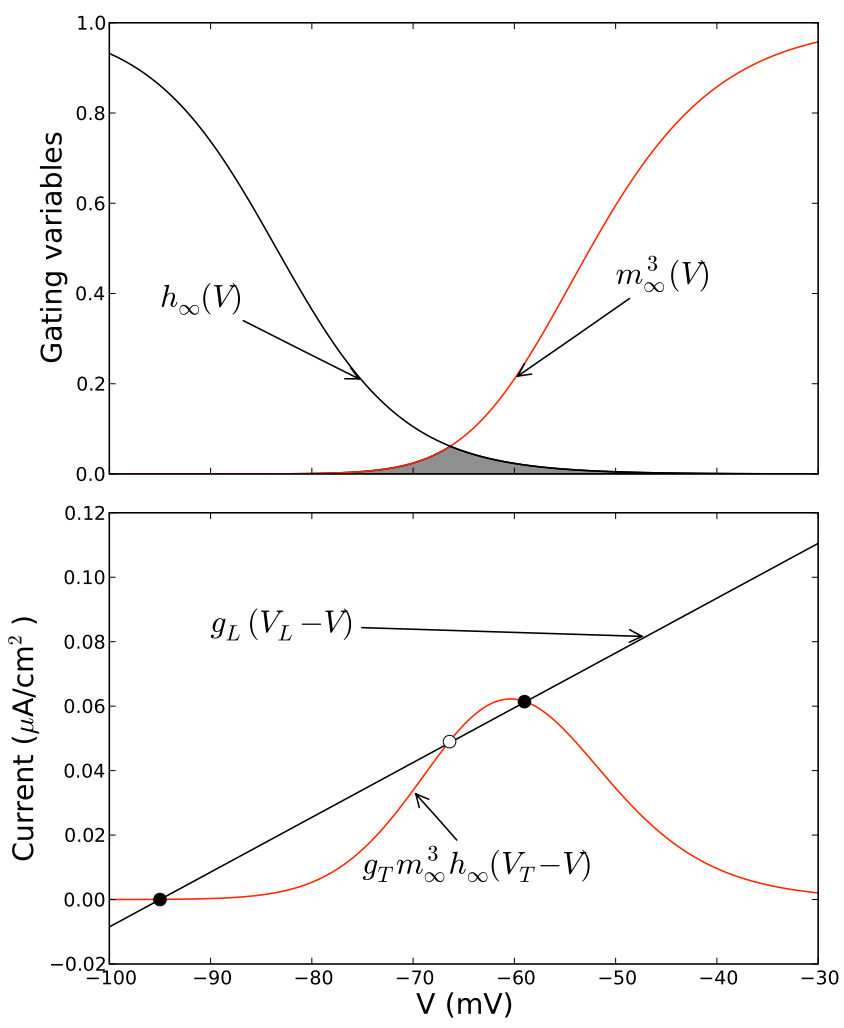

FIG. 1. (Color online) Top: A plot of $m_{\infty}^{3}(v)$ and $h_{\infty}(v)$. The region of overlap defining the voltage range over which the window current is significant is emphasized with the shaded region. Bottom: A plot of the steady state window and leak currents. Equilibria occur where the two curves cross and in this example there are three fixed points. For standard physiological parameters the low- and high-voltage states are stable and the intermediate one is unstable, so that the system is bistable.

$$
\frac{\mathrm{d} x}{\mathrm{~d} t}=\frac{x_{\infty}(v)-x}{\tau_{x}(v)}, \quad x \in\{m, h\},
$$

and the functions for $x_{\infty}(v)$ and $\tau_{x}(v)$ are given in Appendix A. At steady state the $I_{\mathrm{T}}$ current takes the voltage dependent value $I_{\mathrm{T}}^{\infty}(v)=g_{\mathrm{Ca}} m_{\infty}^{3}(v) h_{\infty}(v)\left(v_{\mathrm{Ca}}-v\right)$, which can be visualized using the superposition of the sigmoidal steady-state activation and inactivation curves, as shown in Fig. 1 (top). This diagram nicely illustrates the origin of the phrase "overlap" or "window" current. The voltage range determined by the crossing of the two sigmoids is associated with a sustained current. Hence, although voltage-gated channels are often thought to inactivate (for example, $T$-channels and some $\mathrm{Na}$ channels generate transient currents), a sustained current could be generated in a model with $I_{\mathrm{T}}$ if the steady state membrane potential lies within the 'window', in this case between -80 to $-50 \mathrm{mV}$. For example, making the very reasonable assumption that spiking currents do not strongly affect steady-state properties, then (in the absence of any injected current) equilibria are determined by the condition $g_{\mathrm{L}}\left(v-v_{\mathrm{L}}\right)=I_{\mathrm{T}}^{\infty}(v)$, and as illustrated in Fig. 1 (bottom) there is the potential for bistability. 


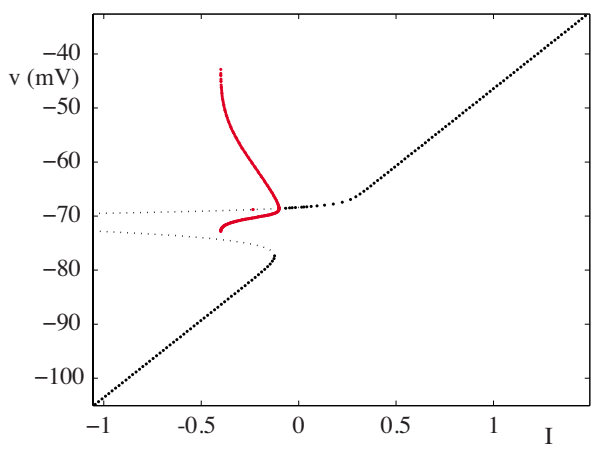

FIG. 2. (Color online) Bifurcation diagram of a nonspiking neuron model with a detailed conductance based model for $I_{\mathrm{T}}$ under constant current injection $I$ (in units of $\mu \mathrm{A} / \mathrm{cm}^{2}$ ). Decreasing the injected current leads to bistability. The more depolarized fixed point destabilizes through a Hopf bifurcation leading to a subthreshold limit cycle. Model parameters are given in Appendix A.

\section{A. Subthreshold dynamics: Bifurcation diagram under constant input}

It is natural to explore bifurcations of the detailed biophysical model using numerical techniques. Focusing for the moment on non-spiking behavior we set $I_{\mathrm{Sp}}=0$ and explore how the fixed point behavior varies with a constant current injection. Depending on the ratio $g_{\mathrm{L}} / g_{\mathrm{Ca}}$ either one or three fixed points can be found. The stability of each fixed point can be computed by examining the eigenvalues of the Jacobian of the system defined by Eqs. (1)-(3). Typically this evaluation is done numerically using a software package such as XPPAUT [20]. In Fig. 2, we show the bifurcation diagram obtained using the parameters given in Appendix A. A Hopf bifurcations occurs at $I \equiv I_{\mathrm{HB}}=-0.1$ and a subthreshold stable limit cycle can be observed for the range $I$ $\in[-0.4,-0.1]$. The limit cycle loses stability upon collision with the separatrix (unstable manifold) of the unstable fixed point. For smaller $g_{\mathrm{L}}$, the Hopf bifurcation observed when injecting hyperpolarizing currents occurs later and a zone of bistability is created. This bistability was originally observed by Williams et al. [4].

\section{B. Spiking dynamics: Grazes and Lyapunov exponents}

With the inclusion of ionic currents that allow for spiking behavior, $I_{\mathrm{Sp}} \neq 0$, we would expect sufficiently strong depolarizing current injection, $I$, to produce spiking behavior. In this case the neuron model would fire in tonic mode and $I_{\mathrm{T}}$ would not play a substantial role in generating spike train patterns. However, if $I$ caused bouts of hyperpolarization, then upon removal of this applied current the build up of $I_{\mathrm{T}}$ could lead to low threshold spike and burst firing. To explore this in more detail we consider a simplified model for $I_{\mathrm{Sp}}$ of integrate-and-fire (IF) type and periodically force it using a periodic input $I(t)=I(t+\Delta)$, where $\Delta$ is the forcing period. We denote this model IF- $I_{\mathrm{T}}$. Whenever the voltage, $v$, reaches a threshold $v_{\text {th }}$ then it is discontinuously reset to the value $v_{\mathrm{r}}$. The set of action potential firing times are defined by

$$
T_{n}=\inf \left\{t \mid v(t) \geq v_{\mathrm{th}} ; t \geq T_{n-1}\right\} .
$$

The generation of firing times is thus specified in terms of the properties of an underlying nonsmooth dynamical system. This means that a stretching of phase space will manifest itself for two nearby trajectories of which only one has sufficient velocity near threshold to guarantee a firing event. A drastic difference in subsequent behavior of the two trajectories would result and gives rise to the notion of a grazing bifurcation. In the IF context a graze can lead to two different types of bifurcation. To see this it is convenient to study the IF trajectory without the reset condition. The first type of bifurcation occurs when there is a tangential intersection of the trajectory with the threshold value such that upon variation of the bifurcation parameter the local maxima of the IF trajectory passes through threshold from above. In this case there is loss of a spike in a nonsmooth fashion. In the second scenario a subthreshold local maxima increases through threshold leading to the creation of a new firing event at some earlier time than usual. For subthreshold trajectories that develop a maxima that grows to touch threshold then a spike may be added into a spike train. Ignoring reset for the moment then a similar mechanism can lead to a spike being subtracted as a maxima decreases through threshold. These mechanisms for changing a spiking pattern are illustrated in the right hand panel of Fig. 8.

To quantify the response of the model to stimulation it is natural to construct the Lyapunov exponents, so that one may readily distinguish aperiodic, periodic and chaotic firing patterns. For smooth dynamical systems of the general form $\dot{Z}$ $\equiv d Z / d t=F(Z), Z \in \mathbb{R}^{N}$, these exponents can be easily constructed by solving the variational problem $\dot{u}=D F(Z) u$, where $D F$ is the Jacobian of the vector field $F$. However, the IF reset mechanism means that one must augment the variational problem with a rule for tracking how perturbations evolve at firing times. The effects introduced by the threshold and reset conditions when disrupting the normal flow have been treated extensively in the literature of impact oscillators [21] and standard IF models [22]. We discuss here briefly the method in the case of the IF- $I_{\mathrm{T}}$ model with $Z$ $=(v, h)$. A firing event can be defined using the indicator function $\mathcal{H}_{\mathrm{th}}(Z)=v-v_{\text {th }}$ and setting $\mathcal{H}_{\mathrm{th}}\left(Z\left(T_{n}\right)\right)=0$. The reset mechanism can then be implemented with $Z^{+}\left(T_{n}\right)$ $=\mathcal{H}_{\mathrm{r}}\left(Z^{-}\left(T_{n}\right)\right)$ where $\mathcal{H}_{\mathrm{r}}(v, h)=\left(v_{\mathrm{r}}, h\right)$. Here the notation $Z^{-}\left(Z^{+}\right)$denotes the trajectory just before (after) firing. By expanding the above equations to leading order one can track how a perturbed trajectory $Z^{-}+\delta Z^{-}$is modified by the firing event to give a post firing trajectory $Z^{+}+\delta Z^{+}$. Namely, we have:

$$
\begin{gathered}
D \mathcal{H}_{\mathrm{th}} \delta \mathrm{Z}^{-}=-D \mathcal{H}_{\mathrm{th}} F\left(Z^{-}\right) \delta t, \\
\delta \mathrm{Z}^{+}=D \mathcal{H}_{\mathrm{r}} \delta \mathrm{Z}^{-}+\left[D \mathcal{H}_{\mathrm{r}} Z^{-}-F\left(Z^{+}\right)\right] \delta t,
\end{gathered}
$$

where $D \mathcal{H}_{\mathrm{th}}\left(D \mathcal{H}_{\mathrm{r}}\right)$ denotes the Jacobian of $\mathcal{H}_{\mathrm{th}}\left(\mathcal{H}_{\mathrm{r}}\right)$. Because the threshold and reset conditions do not depend on $h$, one can see that $\delta t=-\delta v^{-} / \dot{v}^{-}=-\delta v^{+} / \dot{v}^{+}$and hence that $\delta v^{+}$ $=\left(\dot{v}^{+} / \dot{v}^{-}\right) \delta v^{-}$. Therefore, the effect of the discontinuous reset means that, compared with the Lyapunov exponent for smooth dynamics, the perturbed voltage variable needs to be 


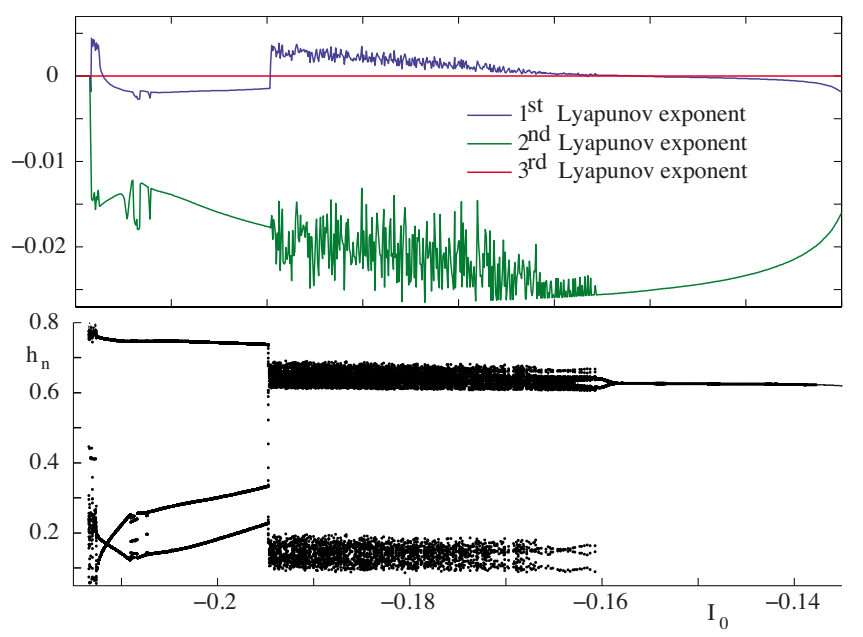

FIG. 3. (Color online) Top: A plot of the Lyapunov exponen $\mathrm{t}$ in the IF- $I_{\mathrm{T}}$ model with square wave periodic forcing. Parameters of the square-wave forcing are $I_{1}=0.5$ and $\Delta=200 \mathrm{~ms}$. Here $v_{\mathrm{r}}=-64 \mathrm{mV}$ and $v_{\mathrm{th}}=-35 \mathrm{mV}$. The largest Lyapunov exponent is positive over the range $I_{0} \in[-0.195,-0.16]$ indicative of chaotic response. Bottom: Value of the deinactivation gating variable $h$ at $V=-70 \mathrm{mV}$. The abrupt transition to a chaotic response at $I_{0}=-0.195$ can be seen as a change in the density of the return value of $h$ (when $v=-60 \mathrm{mV}$ ).

scaled by an amount $\dot{v}^{+} / \dot{v}^{-}$at each firing time.

In Fig. 3, we show the numerically obtained results for the Lyapunov exponents (upper panel) when periodically forcing with a square wave current. This current is such that $I(t)$ $=I_{0}-I_{1}$ if $0 \leq t<\Delta / 2$ and $I(t)=I_{0}+I_{1}$ if $\Delta / 2 \leq t<\Delta$. Along the interval $I_{0} \in[-0.214,-0.136]$, one observes an alternation of regions with a negative or positive maximal Lyapunov exponent. Increasing $I_{0}$, a nonsmooth bifurcation is observed at $I_{0}=-0.195$ resulting in an abrupt change in the dynamics of the model which switches from a periodic to a chaotic response. The region of chaotic dynamics terminates by another nonsmooth bifurcation at $I_{0}=-0.16$. The region of positive maximal Lyapunov exponent in between these two nonsmooth bifurcations gives trajectories which display sensitivity to initial conditions. A typical plot of a chaotic orbit in this region is shown in Fig. 4. We display in the lower

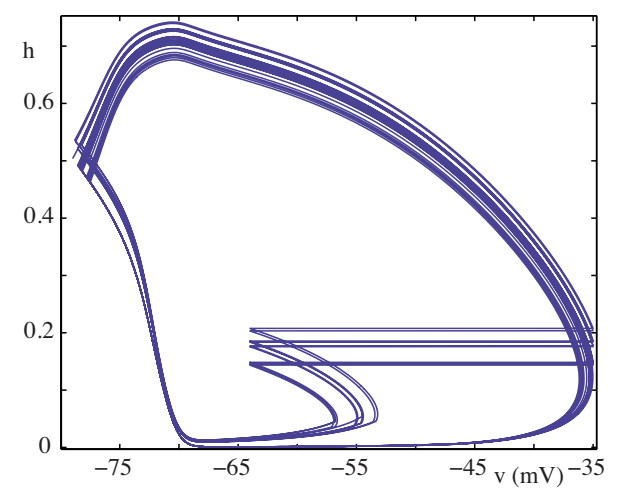

FIG. 4. (Color online) Chaotic trajectory of the IF- $I_{\mathrm{T}}$ model. The figure displays a phase plane view of a spiking chaotic orbit for $I_{0}=-0.175$ and $I_{1}=0.5$. The largest Lyapunov exponent is 0.0025 . panel of Fig. 3 return values of $h$ when $v=-60 \mathrm{mV}$, crossing from below and discarding transients. Numerical simulations show that the second nonsmooth bifurcation occurs close to a subthreshold period doubling bifurcation.

\section{REDUCED MODEL}

To understand the mechanism for generating chaos it is natural to seek a caricature of the $I_{\mathrm{T}}$ current that is analytically tractable. In this spirit Smith et al. have developed the IFB model [11]. In the IFB model the activation variable $m$ is assumed to be fast and is approximated by its steady state value $m_{\infty}(v)$. Moreover, the sigmoidal shape of this function is changed in favor of a Heaviside so that $m_{\infty}(v)=H\left(v-v_{h}\right)$, for some (hyperpolarized) threshold $v_{h}$. The inactivation variable $h$ again has dynamics given by Eq. (3), though with the choice $h_{\infty}(v)=H\left(v_{h}-v\right)$, and $\tau_{h}(v)=\tau_{+} H\left(v_{h}-v\right)+\tau_{-} H(v$ $\left.-v_{h}\right)$. However, the IFB model does not have a steady state calcium current since the activation and inactivation curves are Heaviside functions that switch at the same point $(v$ $\left.=v_{h}\right)$ and $I_{T}^{\infty}(v)=0$ everywhere. As we have linked the presence of chaotic orbits to the existence of a nonzero overlap current we instead seek a model with more generality. With this in mind we imagine decomposing the $(v, h)$ phase space into four regions (as opposed to two separated by the line $v=v_{h}$ for the IFB model). We modify the original IFB model and introduce a PWL activation function to allow for the presence of a nonzero window current and write

$$
m_{\infty}^{3}(v)= \begin{cases}0 & v<v_{1} \\ \left(v-v_{1}\right) /\left(v_{2}-v_{1}\right) & v_{1} \leq v<v_{2} \\ 1 & v>v_{2}\end{cases}
$$

where $v_{1}<v_{h}$ and $v_{2}>v_{h}$. Therefore, in the region $v$ $\in\left[v_{1}, v_{h}\right]$, a steady state calcium current $I_{T}^{\infty}(v)=g_{\mathrm{Ca}}(v$ $\left.-v_{1}\right)\left(v_{\mathrm{Ca}}-v\right) /\left(v_{2}-v_{1}\right)$ exists. A plot of this window current is shown in Fig. 5.

\section{A. Subthreshold behavior of PWL model}

To show that our PWL model of $I_{T}$ does indeed capture the essential behavior of the full model we show the corresponding bifurcation to Fig. 2 in Fig. 6. A comparison of the two shows that there is a good qualitative agreement, both displaying a Hopf bifurcation as the injected current becomes more hyperpolarizing. Both models display a region of co-existence of a subthreshold limit cycle and a fixed point. Moreover, the current value at the Hopf bifurcation is very similar $\left(I_{\mathrm{HB}}=-0.1\right.$ for the full model compared to $I_{\mathrm{HB}}$ $=-0.07$ for the PWL model-see Fig. 6) and quantitatively the main differences are the extent of the region of existence of the limit cycle. However, unlike the bifurcation diagram for the full model that of the simplified model can be calculated analytically. To see how this is achieved we first make use of the fact that the reversal potential for calcium is large (typically $\sim 120 \mathrm{mV}$ ) and make the reasonable approximation $v_{\mathrm{Ca}}-v \approx v_{\mathrm{Ca}}$. We then have that in any given region of phase space the dynamics is governed by a linear system of equations that can be written in the form $\dot{Z}=A Z+b(t)$, where 


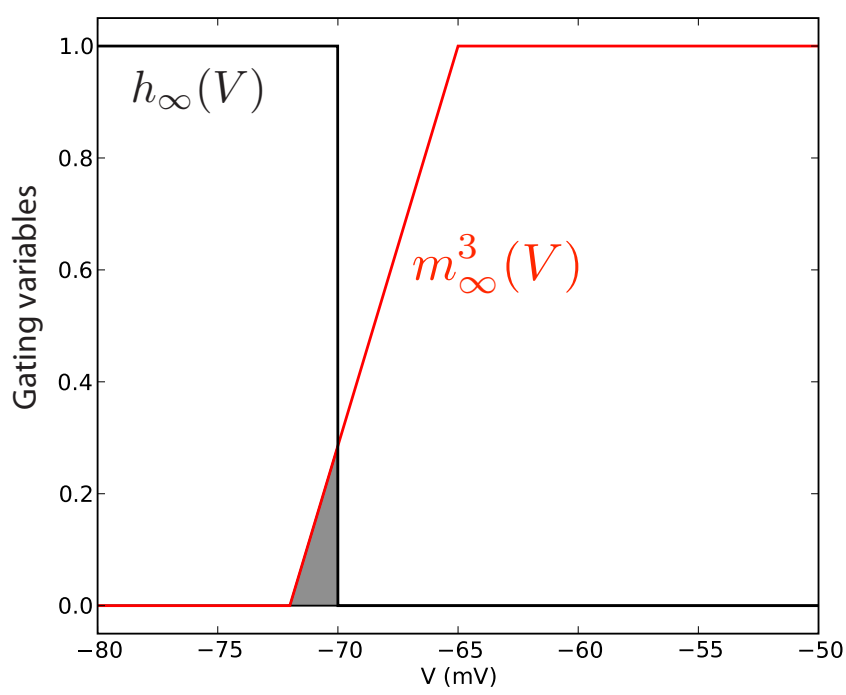

FIG. 5. (Color online) Plot of the PWL activation $m_{\infty}^{3}(V)$ and inactivation function $h_{\infty}(V)$. This reduced model includes a window current and allows for mathematical analysis of the neuron response to forcing.

all voltages are expressed relative to $v_{\mathrm{L}}$. The $2 \times 2$ constant matrix $A$ is readily calculated in the four distinct regions separated by the lines $v=v_{1}, v=v_{2}$, and $v=v_{h}$ (though we do not list the elements here), and $b(t)=(I(t) / C, 0)$. In each of the four regions it is an elementary exercise to calculate fixed points and determine their linear stability. Moreover, it is also possible to construct subthreshold periodic orbits by noting that the dynamics for the gating variable $h$ is either exponentially increasing when $v<v_{h}$ or exponentially decreasing when $v>v_{h}$. Hence, for $t>t_{0}$, we may write:

$$
h(t)= \begin{cases}1+\left[h^{+}\left(t_{0}\right)-1\right] e^{-\left(t-t_{0}\right) / \tau_{+}} & v \leq v_{h} \\ h^{-}\left(t_{0}\right) e^{-\left(t-t_{0}\right) / \tau_{-}} & v \geq v_{h}\end{cases}
$$

where $h^{ \pm}\left(t_{0}\right)$ are the values of $h$ when $v=v_{h}$. The voltage dynamics can then be integrated to obtain

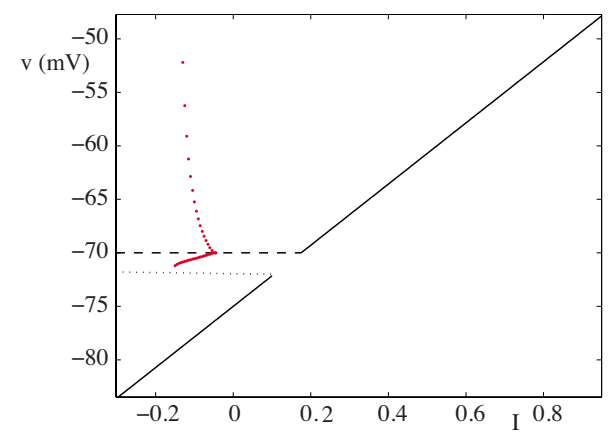

FIG. 6. (Color online) Bifurcation diagram of an IF model with a PWL $I_{\mathrm{T}}$ current under constant current injection $I$ (in units of $\left.\mu \mathrm{A} / \mathrm{cm}^{2}\right)$. The reduced model displays both the bistability and the stable subthreshold orbit which characterized the bifurcation diagram of 2. Models parameters are $v_{1}=-72 \mathrm{mV}, v_{h}=-70 \mathrm{mV}$, and $v_{2}=-65 \mathrm{mV}$. The maximal conductance constants are identical to the full $I_{\mathrm{T}}$ model.

$$
v(t)=v_{\mathrm{L}}+G\left(t, t_{0}\right) v\left(t_{0}\right)+\int_{t_{0}}^{t} G(t, s) J(s) d s,
$$

where

$$
J(t)=I(t) / C+ \begin{cases}0 & v<v_{1} \\ -v_{1} h(t) / \hat{\tau}_{\mathrm{Ca}} & v_{1} \leq v \leq v_{2} \\ v_{\mathrm{Ca}} h(t) / \tau_{\mathrm{Ca}} & v>v_{2}\end{cases}
$$

and $G(t, s)=\exp \int_{s}^{t} K\left(s^{\prime}\right) d s^{\prime}$, with

$$
K(t)=\left\{\begin{array}{ll}
-1 / \tau & v<v_{1} \text { and } v>v_{2} \\
-1 / \tau+v_{\mathrm{Ca}} h(t) / \tau_{\mathrm{Ca}} & v_{1} \leq v \leq v_{2}
\end{array},\right.
$$

where $\tau=C / g_{\mathrm{L}}, \quad \tau_{\mathrm{Ca}}=C / g_{\mathrm{Ca}}, \quad$ and $\quad \hat{\tau}_{\mathrm{Ca}}=\tau_{\mathrm{Ca}}\left(v_{2}-v_{1}\right) / v_{\mathrm{Ca}}$. Hence, trajectories can be constructed in closed form using Eqs. (8) and (9) from a given set of initial data and patching solutions (enforcing continuity) at the phase space boundaries where $v=v_{1}, v=v_{h}$ and $v=v_{2}$. For example, a simple periodic orbit with $v(t) \in\left(v_{1}, v_{2}\right)$ of period $\Delta$ that crosses through $v=v_{h}$ just twice can be specified by solving $v\left(t_{1}\right)=v_{h}$ using initial data $\left(v_{h}, h_{0}\right)$, and $v\left(t_{2}\right)=v_{h}$ with initial data $\left(v_{h}, h\left(t_{1}\right)\right)$. The unknown value for $h_{0}$ is determined self-consistently by enforcing periodicity of $h$, namely that $h\left(t_{1}+t_{2}\right)=h_{0}$. The simultaneous solution of these three equations gives the period as $\Delta=t_{1}+t_{2}$. The stability of the orbit can be determined using Floquet theory for PWL systems as recently developed in [23]. Using this approach we find that the nonzero Floquet exponent is given explicitly by

$$
\sigma=-\frac{t_{1}}{\tau_{-}}-\frac{t_{2}}{\tau_{+}}-\frac{\Delta}{\tau}+\frac{1}{\hat{\tau}_{\mathrm{Ca}}} \int_{0}^{\Delta} h(t) d t .
$$

Periodic solutions are stable if $\sigma<0$, and this condition was used to determine the stability of solution branches in the bifurcation diagram of Fig. 6.

\section{B. Spiking behavior of PWL model}

The spiking behavior of the PWL model is also consistent with that of the full model and supports chaotic trajectories. An example chaotic trajectory is shown in Fig. 7. Once again it is possible to obtain trajectories in closed form. Indeed these are also given by Eq. (9) subject to the further condition that $v$ is reset to $v_{\mathrm{r}}$ if $v=v_{\mathrm{th}}$. Since direct numerical simulations suggest that chaotic orbits are organized around unstable periodic orbits it is natural to consider the construction of mode-locked states. To do this we follow along similar lines to [12] and look for periodic spike trains with period $q \Delta$, which can be written in the form $T_{n}=\left([n / p]+\phi_{n(p)}\right.$ $\left.-\phi_{h}\right) q \Delta$, where $\phi_{h}$ is the phase at which the neuron crosses $v_{h}$ from below and $\phi_{n(p)}$ the set of $p$ phases at which the neuron spikes. Here, [.] denotes the integer part and $n(p)$ $=n \bmod p$. In addition to being interested in the set of spike times we are now also concerned in tracking the set of times $B_{n}=\inf \left\{t \mid v(t) \geq v_{h}, \dot{v}>0, t>B_{n-1}\right\}$ where $v_{h}$ is crossed from below. Taking for example a simple 1:1 spiking orbit, we denote $T_{1}=t_{1}$ and $B_{1}=t_{2}$. We can specify the trajectory by $v\left(t_{1}\right)=v_{\text {th }}$ with initial data $\left(v_{h}, h_{0}\right), v\left(t_{2}\right)=v_{h}$ with initial data $\left(v_{\mathrm{r}}, h\left(t_{1}\right)\right)$ and enforcing $h(\Delta)=h_{0}$, with $\Delta=t_{1}+t_{2}$. In practice, 


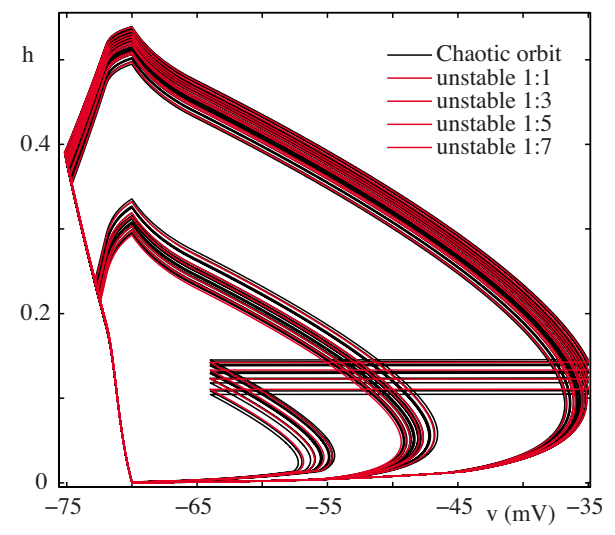

FIG. 7. (Color online) Phase-plane of a spiking chaotic orbit (black line). At least 4 unstable orbits (red lines) are embedded in this chaotic attractor. A detailed view of the trajectory and unstable periodic orbits is displayed in Fig. 8. Model parameters are the same as in Fig. 6 with $v_{\mathrm{r}}=-64 \mathrm{mV}$ and $v_{\mathrm{th}}=-35 \mathrm{mV}$.

any periodic solution can be written in a similar fashion, enforcing the constraint on $h$ at the crossing of $v_{h}$ and patching together trajectories (along the lines described in Sec. III A) subject to reset. More elaborate $p: q$ solutions ( $p$ spikes per $q$ cycles of firing) can be constructed in a similar fashion. Every time that a trajectory crosses a border $v_{\mathrm{B}}$ $\in\left\{v_{1}, v_{2}, v_{h}, v_{\text {th }}\right\}$ we have a constraint equation of the form $v\left(t_{\mu}\right)=v_{\mathrm{B}}$, which determines a "time-of-flight" $t_{\mu}$. Each of these constraint equations can be written in the general form $F\left(t_{\mu+1}, t_{\mu}, h_{\mu}\right)=0$, where the function $F$ is given by Eq. (9) evaluated at $t=t_{\mu+1}$ starting from $t_{0}=t_{\mu}$. Here $h_{\mu}$ is the value of $h$ when $v$ most recently crossed $v=v_{h}$, namely $h\left(\sum_{\nu=1}^{\mu} t_{\nu}\right)$ $=h_{\mu}$. This latter expression can also be written as a constraint of the form $G\left(t_{\mu+1}, t_{\mu}, h_{\mu}\right)=h_{\mu+1}$, where the function $G$ is given by Eq. (8) with $t=t_{\mu+1}, t_{0}=t_{\mu}$ and $h\left(t_{0}\right)=h_{\mu}$. Any trajectory is thus determined by a set of $2 \mu$ nonlinear equations:

$$
F\left(t_{\mu+1}, t_{\mu}, h_{\mu}\right)=0, \quad G\left(t_{\mu+1}, t_{\mu}, h_{\mu}\right)=h_{\mu+1} .
$$

Introducing perturbations (to the periodic orbit) of the form $\left(t_{\mu}, h_{\mu}\right) \rightarrow\left(t_{\mu}, h_{\mu}\right)+\left(\delta t_{\mu}, \delta h_{\mu}\right)$ gives:

$$
\begin{gathered}
\delta t_{\mu+1} \frac{\partial F}{\partial t_{\mu+1}}+\delta t_{\mu} \frac{\partial F}{\partial t_{\mu}}+\delta h_{\mu} \frac{\partial F}{\partial h_{\mu}}=0 \\
\delta t_{\mu+1} \frac{\partial G}{\partial t_{\mu+1}}+\delta t_{\mu} \frac{\partial G}{\partial t_{\mu}}+\delta h_{\mu} \frac{\partial G}{\partial h_{\mu}}=\delta h_{\mu+1} .
\end{gathered}
$$

The above may be rearranged to give the linearized equations of motion in the form $\gamma_{\mu+1}=M_{\mu} \gamma_{\mu}$, where $\gamma_{\mu}$ $=\left(\delta t_{\mu}, \delta h_{\mu}\right)$ and the matrix $M_{\mu}$ is given in Appendix B. Hence, for a periodic orbit written in terms of $p$ 'patched' trajectories the matrix determining stability is $\Lambda=M_{p} M_{p-1} \ldots M_{2} M_{1}$. If the two eigenvalues of $\Lambda$ lie within the unit circle then the orbit is stable. Stable orbits can loose stability through saddle-node, period-doubling or NeimarkSacker bifurcations.

We show in Fig. 7 a chaotic trajectory of the IF- $I_{\mathrm{T}}$ model with a PWL model of $I_{T}$. This trajectory is similar to that observed for the full $I_{\mathrm{T}}$ model. By tracking unstable mode-
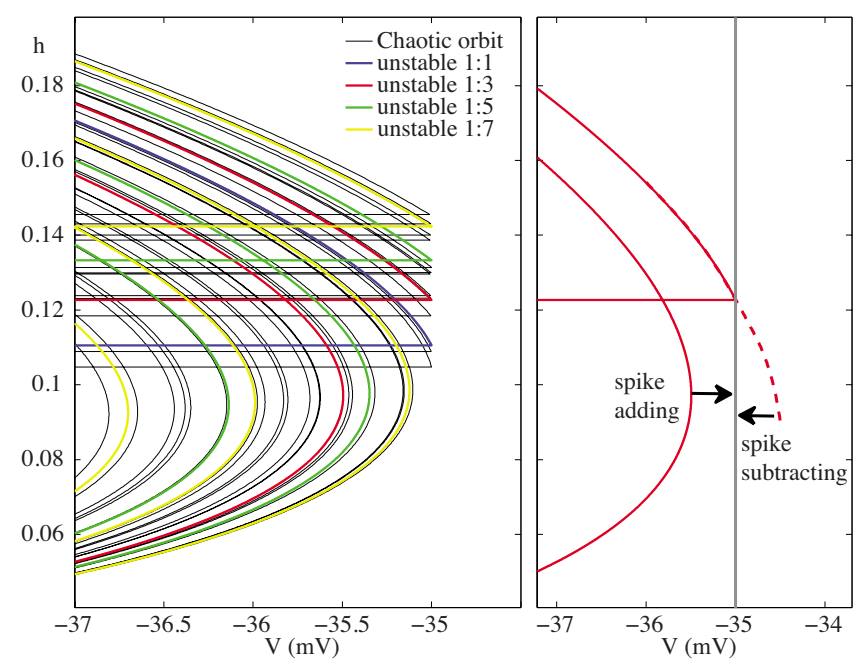

FIG. 8. (Color online) Left, Detailed plot of the chaotic trajectory and unstable mode-locked solutions near threshold. Each unstable mode-locked solution is plotted with the $1: 1$ in blue, $3: 1$ in red, 5:1 in green, and the 7:1 in yellow. Right, Example of the spike adding and spike subtracting mechanism for a 3:1 mode. The dotted line represents the unrestricted trajectory (i.e., no reset).

locked orbits in parameter space we were able to establish that they coexist with the chaotic orbit. In Fig. 7, we show four such unstable modes with $q=1$ and $p \in\{1,3,5,7\}$. We found only modes of the form $(2 m+1): 1$ where $m \in \mathbb{N}$. The reason for this lies in the amount of de-inactivation that can be reached after a spike. Indeed, a spike occurs at the beginning of a cycle (i.e., when $\left.I(t)=I_{0}+I_{1}\right)$ when the $\mathrm{Ca}^{2+}$ channels are sufficiently deinactivated. More precisely, $h$ needs to be increased high enough during the hyperpolarizing part of the period [i.e., when $I(t)=I_{0}-I_{1}$ ] for a spike to occur. After a reset, groups of two subthreshold cycles, one small amplitude and the other large amplitude, are necessary before threshold can once again be reached. Numerical simulation clearly shows that chaotic trajectories are organized around unstable periodic mode-locked solutions and are shaped by spike adding or spike subtracting mechanisms as described in Sec. II B. Indeed, when a trajectory is started close to an unstable mode-locked state, it diverges from it quickly and evolves toward an orbit that can graze the threshold. As an example, we now describe what happens for a trajectory initiated close to a 3:1 mode. Initially the trajectory spikes once every three forcing periods, tracking near to the 3:1 solution. If we denote by $h_{n}$ the value of $h$ at the $n$th threshold crossing and by $h_{3: 1}^{*}$ the value of $h$ at threshold for the 3:1 mode, our numerical simulations show that $h_{n}$ then begins to oscillate around $h_{3: 1}^{*}$ taking subsequently higher and lower values. The group of subthreshold cycles following a threshold crossing with $h_{n}>h_{3: 1}^{*}$ has a maximum membrane potential that approaches $v_{\text {th }}$ from below. This can ultimately lead to a spike addition via the mechanism described in Sec. II B, and illustrated in the right hand panel of Fig. 8. The group of subthreshold cycles following a threshold-crossing with $h_{n}$ $<h_{3: 1}^{*}$ have a smaller and smaller $\dot{v}$ at $v=v_{\text {th }}$ and ultimately suffer a spike subtraction. We further observed that a trajectory initially close to the $3: 1$ orbit could make a transition to an orbit close to the 5:1 orbit. In general, we expect that the 
loss or addition of a spike (via a graze of the trajectory with the firing threshold), can cause a transition from a trajectory organized around one unstable $p: q$ mode-locked state to another (with a different set of $(p, q)$ values). An example of such a scenario is shown in Fig. 8 (left) with a portion of a chaotic orbit shown in relation to the unstable mode-locked states with $q=1$ and $p \in\{1,3,5,7\}$. In the next section we show how a construction of the unstable periodic orbits can be combined with the grazing condition (for spike adding and subtracting) to determine an effective one-dimensional map that captures the essentials of the observed chaotic behavior.

\section{One-dimensional map}

There are many incentives to use a dimensionally reduced description to describe the observed chaotic behavior. One of these is the desire to understand the organization of trajectories in terms of grazes which add or subtract spikes. In the context of impact oscillators grazing bifurcations are well known to allow an effective description of system dynamics, including chaotic trajectories, in terms of a discontinuous one-dimensional map [18]. Because of the presence of grazing bifurcations in the IF- $I_{\mathrm{T}}$ there is some hope of mirroring the success of the impact oscillator community [24,25] in this regard, especially if we focus on the tractable PWL model of $I_{\mathrm{T}}$.

We construct a one-dimensional map $\mathcal{R}$ giving the return values $h_{n}$ of the slow variable $h$ at the spike time $T_{n}$. Each $p: q$ mode-locked solution of the periodically forced $\mathrm{IF}-I_{\mathrm{T}}$ corresponds to a fixed point of the map $\mathcal{R}^{(p)}$. Here we shall focus on the $(2 m+1): 1$ modes and begin by calculating the fixed points $h_{(2 m+1): 1}^{*}$ of $h_{n+1}=\mathcal{R}^{(2 m+1)}\left(h_{n}\right)$. These are obtained by solving the set of nonlinear equations given by Eq. (13). We can use the 'patched' dynamics in order to approximate the behavior of $\mathcal{R}$ near the fixed points. Looking at the first order perturbation in $h_{n}$ of Eq. (13) we can obtain the slope of $\mathcal{R}$ at each fixed point: $d \mathcal{R} /\left.d h\right|_{h=h^{*}}=[\Lambda]_{12}$. We now split the domain of $\mathcal{R}$ into different intervals. Each interval corresponds to all possible $h_{n}$ values taken by an orbit initially close to a $(2 m+1): 1$ mode before undergoing a graze. Hence, we determine the boundaries of each interval by computing the values $A_{2 m+1}$ of $h$ where a spike addition occurs. Namely, $\left\{h_{g}^{ \pm}=h\left(T_{n}\right) \mid \dot{v}\left(T_{n}\right)=0\right\}$ where the $g$ subscript indicates that we are at grazing and the + and - superscripts indicates a spike addition and subtraction, respectively. For example, the interval $I_{(2 m+1): 1}$ of $h_{n}$ values attainable by an orbit initially close to a $(2 m+1): 1$ mode is given by $I_{(2 m+1): 1}=\left[h_{g(2 m+1): 1}^{+}, h_{g(2 m+1): 1}^{-}\right)$. To compute $h_{g(2 m+1): 1}^{ \pm}$, we assume that the phases $\phi_{n}$ are roughly those of the $(2 \mathrm{~m}$ $+1): 1$ mode as motivated by numerical simulations. Then, we solve Eqs. (9) and (8) using the necessary conditions for each type of graze. In practice, one can see that we should have $h_{g(2 m+1): 1}^{-}=h_{g(2 m+3): 1}^{+}$for the map to be defined on a continuous domain. Thus we need only calculate $h_{g(2 m+1): 1}^{-}$. Examples of values $h_{g(2 m+1): 1}^{ \pm}$are given in Fig. 9, with spike addition represented by a square marker, labeled by $A_{1,3,5,7}$, and spike subtraction by a diamond marker, labeled by $S_{1,3,5,7}$. Just before a spike addition, the return value of $\mathcal{R}$ is

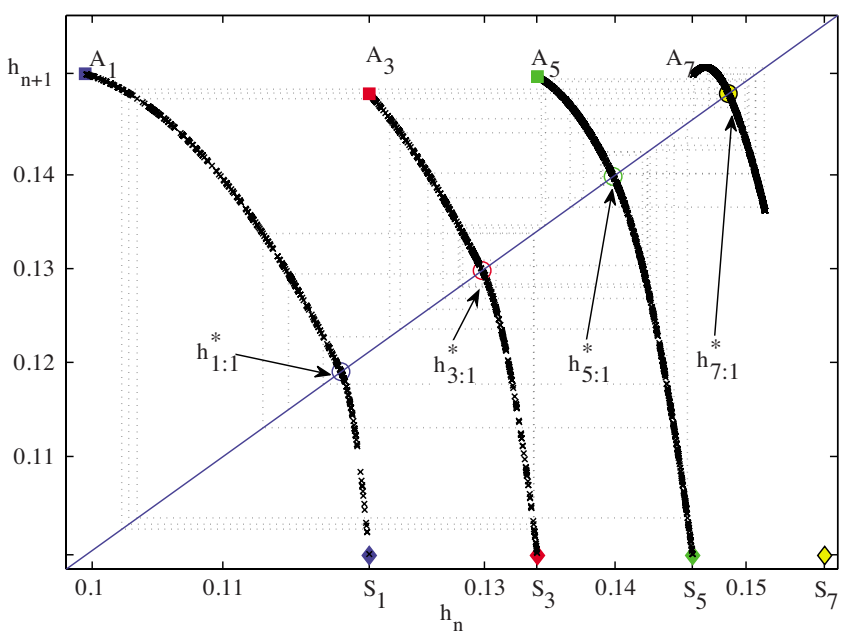

FIG. 9. (Color online) One-dimensional map approximation. The unstable fixed points of the return map correspond to the value of $h$ at $v=v_{\text {th }}$ for the $1: 1,3: 1,5: 1$, and 7:1 unstable mode-locked solutions. Each branch is defined over a range whose borders correspond to the value of $h_{n}$ for which a grazing bifurcation occurs.

maximal on the interval $I_{(2 m+1): 1}$. Similarly, the minimal return value on the interval $I_{(2 m+1): 1}$ occurs just before a spike subtraction. These maximal and minimal values are important features of $\mathcal{R}$ since they determine the range of $\mathcal{R}$ on each interval $\left[h_{g(2 m+1): 1}^{+}, h_{g(2 m+1): 1}^{-}\right)$. Finally, as a minimal map that incorporates all these constraints, we implement a second-order polynomial interpolation between $S_{i}, A_{i}$ and $h_{i: 1}^{*}$. The return map has the form $\mathcal{R}: h \rightarrow a^{+} h^{2}+b^{+} h+c^{+}$for each interval $\left[h_{g}^{+}, h^{*}\right)$ and $\mathcal{R}: h \rightarrow a^{-} h^{2}+b^{-} h+c^{-}$for each interval $\left[h^{*}, h_{g}^{-}\right)$with the coefficients of the polynomial interpolation given in Appendix C. Figure 9 shows the onedimensional map approximation obtained by implementing the above method for $I_{0}=-0.175$ and $I_{1}=0.5$ (where chaotic behavior in the original system occurs). Orbits of the reduced map and sections from the full dynamics may be easily compared. In Fig. 10, we show the corresponding histograms of $h_{n}$ values for the full and reduced descriptions, illustrating that using the one-dimensional map is indeed an excellent way to characterize the chaotic behavior of a fast spiking neuron model with a slow $T$-type calcium current.

\section{DISCUSSION}

We have shown in this paper how the $I_{\mathrm{T}}$ current present in thalamic neurons can be approximated by an (analytically tractable) PWL caricature. The subsequent generation of a "window" current, known to be at the origin of the bistability of the membrane potential of thalamic neurons, was shown to be responsible for the existence of chaotic trajectories. Using closed form solutions for trajectories of an IF model with this current we were able to calculate the periodic orbits of the homogeneous (i.e., subthreshold limit cycle) and periodically forced system. Importantly we have shown that unstable mode-locked solutions constitute an important set of organizing centers for chaotic trajectories, and have used them, in conjunction with grazing bifurcations, to build a reduced one-dimensional map. 


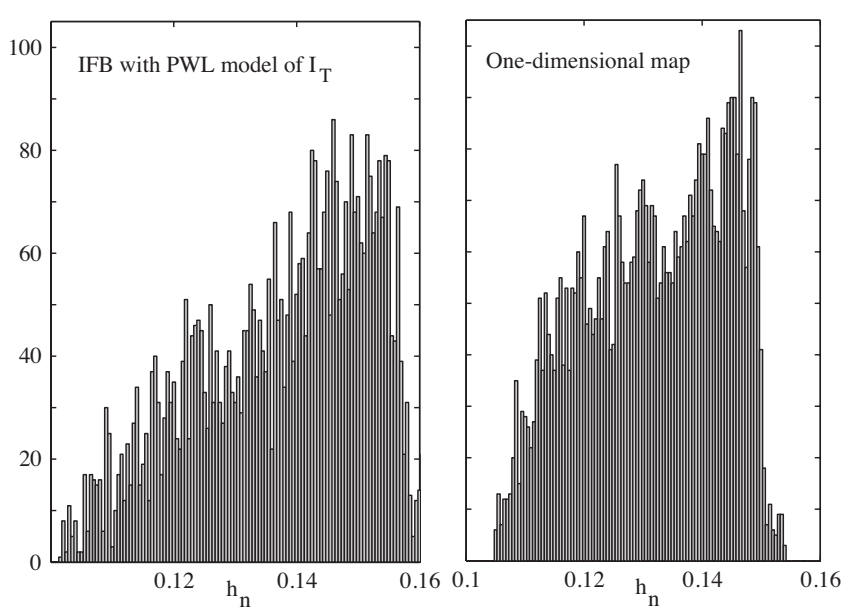

FIG. 10. Density of return values for $h$ obtained for the PWL IF- $I_{\mathrm{T}}$ model and for the one-dimensional map derived using the construction of unstable mode-locked orbits and grazing bifurcations.

The one-dimensional map we obtained bears some resemblance to that derived by Nordmark [18] for an impacting mechanical oscillator. The derivation of this mechanical impact map relies on the existence of orbits with a large number of nonimpacting cycles before crossing some phase space discontinuity. In this case there is the possibility of a specific bifurcation characterized by a period-adding sequence interleaved by chaotic windows. In our case, the number of subthreshold orbits is rather small (0 to 6), and there are no stable periodic orbits in the chaotic region. Interestingly, the different branches of our one-dimensional map describe sequences of $(2 m+1): 1$ periodic orbits rather than a periodadding between $n: 1$ periodic orbits. Hopefully the study of other forced single neuron models of IF type, such as the quadratic IF model [26], the adaptive exponential IF model [27], the absolute IF model [28] and the Izhikevich model [29] may all benefit by a further translation of ideas from the impact oscillator literature. Perhaps more important though is the extension of this work to the network level. Matveev et al. [30] have already analyzed anti-phase bursting in a pair of reciprocally interacting spiking cells with synaptic inhibition and a slow $T$-type calcium current. In this case the single neuron model did not possess an overlap current and only periodic bursting patterns were observed. It thus becomes an interesting question as to whether with the inclusion of overlap currents chaotic responses can be enhanced or suppressed by synaptic coupling. For the study of larger networks with overlap currents one can generalize the approach in [15] which constructs firing rate models from spiking models in the presence of slow intrinsic and synaptic currents. In this case the firing rate function that mediates interactions depends on both the synaptic drive and the slow intrinsic current. For a $T$-type calcium current without overlap rhythms and waves have already been analyzed and it would be natural to extend these calculations to the piece-wise linear model of $I_{\mathrm{T}}$ introduced in this paper. Moreover, as such networks (with minor refinement to distinguish between thalamo-cortical and reticular cell types) can provide a mini- mal model of the thalamus it becomes even more interesting to analyze the network response to periodic forcing, and to determine the conditions for mode-locked behavior. This is especially relevant to understanding how cortical population responses arise from sensory input (gated through the thalamus) and the dependence of this response on thalamocortical interactions and intrinsic currents. This is a topic of current study and will be reported on elsewhere.

\section{ACKNOWLEDGMENTS}

J.L. is supported by the European Commission (EC Contract No. MEST-CT-2005-020723). Chris Sumner was supported by the Medical Research Council (U.K.).

\section{APPENDIX A}

The conductance based model for $I_{\mathrm{T}}$ is given by Eqs. (2) and (3) with

$$
\begin{gathered}
m_{\infty}(v)=\frac{1}{\left.1+e^{-(v+72) \beta 3}\right)}, h_{\infty}(v)=\frac{1}{1+e^{2(v+70)}}, \\
\tau_{h}(v)=7.66+0.02868 e^{-0.1054 v},
\end{gathered}
$$

and $v_{\mathrm{L}}=-95.0 \mathrm{mV}, g_{\mathrm{Ca}}=0.7 \mathrm{~m} \mathrm{~S} \mathrm{~cm}^{-2}$ and $v_{\mathrm{T}}=120 \mathrm{mV}$. Other parameters in Eq. (1) are $C=2 \mu \mathrm{F} \mathrm{cm}^{-2}$ and $g_{\mathrm{L}}$ $=0.35 \mathrm{~m} \mathrm{~S} \mathrm{~cm}^{-2}$.

\section{APPENDIX B}

The elements, $\left[M_{\mu}\right]_{i j}$, of $M_{\mu}$ are

$$
\begin{gathered}
{\left[M_{\mu}\right]_{11}=-\frac{\partial F}{\partial t_{\mu}} / \frac{\partial F}{\partial t_{\mu+1}},} \\
{\left[M_{\mu}\right]_{12}=-\frac{\partial F}{\partial h_{\mu}} / \frac{\partial F}{\partial h_{\mu+1}},} \\
{\left[M_{\mu}\right]_{21}=\left[M_{\mu}\right]_{11} \frac{\partial G}{\partial t_{\mu+1}}+\frac{\partial G}{\partial t_{\mu}},} \\
{\left[M_{\mu}\right]_{22}=\left[M_{\mu}\right]_{12} \frac{\partial G}{\partial t_{\mu+1}}+\frac{\partial G}{\partial h_{\mu}},}
\end{gathered}
$$

and are readily obtained by differentiating Eqs. (8) and (9).

\section{APPENDIX C}

$$
\begin{gathered}
a^{ \pm}=\frac{\left(\mathcal{R}\left(h_{g}^{ \pm}\right)+\left(\Lambda_{12}-1\right) h_{g}^{ \pm}+\Lambda_{12} h^{*}\right)}{2\left(h^{*}-h_{g}^{ \pm}\right)^{2}}, \\
b^{ \pm}=\Lambda_{12}-2 a^{ \pm} h^{*}, \\
c^{ \pm}=h^{*}\left(1-\Lambda_{12}+a^{ \pm} h^{*}\right),
\end{gathered}
$$

where $h_{g}^{ \pm}=h_{g(2 m+1): 1}^{ \pm}, h^{*}=h_{(2 m+1): 1}^{*}$ with $\Lambda_{12}$ identified as the slope of the return map $\mathcal{R}$ at a fixed point. 
[1] M. Steriade, Neuronal Substrates of Sleep and Epilepsy (Cambridge University Press, Cambridge, England, 2003).

[2] R. Llinás and H. Jahnsen, Nature (London) 297, 406 (1982).

[3] H. Jahnsen and R. Llinás, J. Physiol. 349, 227 (1984) [http:// www.ncbi.nlm.hih.gov/pmc/articles/PMC1199335].

[4] S. R. Williams, T. I. T'oth, J. P. Turner, S. W. Hughes, and V. Crunelli, J. Physiol. 505, 689 (1997).

[5] S. W. Hughes, D. W. Cope, T. I. T'oth, S. R. Williams, and V. Crunelli, J. Physiol. 517, 805 (1999).

[6] S. W. Hughes, D. W. Cope, K. L. Blethyn, and V. Crunelli, Neuron 33, 947 (2002).

[7] V. Crunelli, T. I. T'oth, D. W. Cope, K. Blethyn, and S. W. Hughes, J. Physiol. 562, 121 (2004).

[8] X. J. Wang, J. Rinzel, and M. A. Rogawski, J. Neurophysiol. 66, 839 (1991).

[9] J. Huguenard and D. A. McCormick, Electrophysiology of the Neuron (Oxford University Press, New York, 1994).

[10] M. E. Rush and J. Rinzel, Biol. Cybern. 71, 281 (1994).

[11] G. D. Smith, C. L. Cox, S. M. Sherman, and J. Rinzel, J. Neurophysiol. 83, 588 (2000).

[12] S. Coombes, M. R. Owen, and G. D. Smith, Phys. Rev. E 64, 041914 (2001).

[13] N. A. Lesica and G. B. Stanley, J. Neurosci. 24, 10731 (2004).

[14] B. Babadi, J. Comput. Neurosci. 18, 229 (2005).

[15] S. Coombes, Phys. Rev. E 67, 041910 (2003).
[16] M. A. Huertas, J. R. Groff, and G. D. Smith, J. Comput. Neurosci. 19, 147 (2005).

[17] M. A. Huertas and G. D. Smith, J. Comput. Neurosci. 21, 171 (2006).

[18] A. B. Nordmark, Phys. Rev. E 55, 266 (1997).

[19] A. L. Hodgkin and A. F. Huxley, J. Physiol. 116, 449 (1952).

[20] B. Ermentrout, Simulating, Analyzing, and Animating Dynamical Systems: A Guide to Xppaut for Researchers and Students (Society for Industrial \& Applied Mathematics, Philadelphia, 2002).

[21] P. C. Müller, Chaos Solitons Fractals 5, 1671 (1995.

[22] S. Coombes, Phys. Lett. A 255, 49 (1999).

[23] S. Coombes, SIAM J. Appl. Dyn. Syst. 7, 1101 (2008).

[24] M. di Bernardo, C. J. Budd, and A. R. Champneys, Phys. Rev. Lett. 86, 2553 (2001).

[25] J. de Weger, D. Binks, J. Molenaar, and W. van de Water, Phys. Rev. Lett. 76, 3951 (1996).

[26] P. E. Latham, B. J. Richmond, P. G. Nelson, and S. Nirenberg, J. Neurophysiol. 83, 808 (2000).

[27] R. Brette and W. Gerstner, J. Neurophysiol. 94, 3637 (2005).

[28] S. Coombes and M. Zachariou, Coherent Behavior in Neuronal Networks (Springer, New York, 2009).

[29] E. M. Izhikevich, IEEE Trans. Neural Netw. 14, 1569 (2003).

[30] V. Matveev, A. Bose, and F. Nadim, J. Comput. Neurosci. 23, 169 (2007). 